H. Fujimoto

Nagoya Math. J.

Vol. 71 (1978), 25-41

\title{
REMARKS TO THE UNIQUENESS PROBLEM OF MEROMORPHIC MAPS INTO $P^{N}(C)$, II
}

\author{
HIROTAKA FUJIMOTO
}

\section{§1. Introduction}

In [7], R. Nevanlinna gave the following uniqueness theorem of meromorphic functions as an improvement of a result of G. Pólya ([8]).

THEOREM A. Let $f, g$ be non-constant meromorphic functions on $\boldsymbol{C}$. If there are five mutually distinct values $a_{1}, \cdots, a_{5}$ such that $f^{-1}\left(a_{i}\right)=$ $g^{-1}\left(a_{i}\right)(1 \leqq i \leqq 5)$, then $f \equiv g$.

The author attempted to generalize this to the case of meromorphic maps of $C^{n}$ into $P^{N}(C)$ and obtained some results in the previous papers [4], [5] and [6]. One of them is the following;

THEOREM B. Let $f$ and $g$ be meromorphic maps of $C^{n}$ into $P^{N}(C)$ one of which is algebraically non-degenerate. If there are $2 \mathrm{~N}+3$ hyperplanes $H_{i}(1 \leqq i \leqq 2 N+3)$ in general position such that $\nu\left(f, H_{i}\right)=$ $\nu\left(g, H_{i}\right)$ for pull-backs $\nu\left(f, H_{i}\right), \nu\left(g, H_{i}\right)$ of the divisors $\left(H_{i}\right)$ by $f$ and $g$ respectively, then $f \equiv g$.

Relating to this, the following theorem will be proved.

THEOREM I. Let $f, g$ be algebraically non-degenerate meromorphic maps of $C^{n}$ into $P^{N}(C)$. If there are hyperplanes $H_{i}$ in general position such that

$$
\nu\left(f, H_{i}\right)=\nu\left(g, H_{i}\right)=0,
$$

namely, $f\left(C^{n}\right) \cap H_{i}=g\left(C^{n}\right) \cap H_{i}=\phi$ for $i=1,2, \cdots, N+1$ and

$$
\min \left(\nu\left(f, H_{j}\right), N\right)=\min \left(\nu\left(g, H_{j}\right), N\right)
$$

for $j=N+2, \cdots, 2 N+3$, then $f \equiv g$.

Received May 19, 1977. 
This will be given as a consequence of the following generalization of a classical result of $R$. Nevanlinna ([7], Satz 7, p. 388).

THEOREM II. Let $f, g$ be algebraically non-degenerate meromorphic maps of $C^{n}$ into $P^{N}(C)$. If there are $N+2$ hyperplanes in general position such that

$$
\nu\left(f, H_{i}\right)=\nu\left(g, H_{i}\right)=0
$$

for $i=1,2, \cdots, N+1$ and

$$
\min \left(\nu\left(f, H_{N+2}\right), N\right)=\min \left(\nu\left(g, H_{N+2}\right), N\right),
$$

then $f$ and $g$ are related as $L \cdot g=f$ with a projective linear transformation $L$ of $P^{N}(C)$ which permutes hyperplanes $H_{1}, \cdots, H_{N+1}$ and leaves $H_{N+2}$ fixed.

In $\S 2$, we shall give a combinatorial lemma which plays an essential role in this paper. In $\S 3$, we shall recall some classical results in the value distribution theory for holomorphic maps of $C$ into $P^{N}(C)$ and obtain a new result from them. Theorems I and II are completely proved in $\S 4$.

\section{§ 2. Main Lemma}

For later use, we shall give in this section a graph-theoretic combinatorial lemma. We consider a set $A=\left\{a_{i j} ; 1 \leqq i \leqq n, 1 \leqq j \leqq n\right\}$ consisting of $n^{2}$ elements abstractly. Let non-empty subsets $C$ of $A$ and $\Gamma$ of $C \times C$ be given in some manner. For any $a_{i j}, a_{k \ell}$ in $C$, we write

$$
(i, j) \leftrightarrow(k, \ell), \quad \text { or } \quad(i, j) \leftrightarrow(k, \ell)
$$

if $\left(a_{i j}, a_{k \ell}\right) \in \Gamma$, or $\left(a_{i j}, a_{k \ell}\right) \notin \Gamma$ respectively. We assume that

$\left(\mathrm{A}_{0}\right)$ for any $a_{i j}, a_{k \ell}$ in $C(i, j) \leftrightarrow(i, j)$ and $(i, j) \leftrightarrow(k, \ell)$ whenever $(k, \ell) \leftrightarrow(i, j)$,

$\left(\mathrm{A}_{1}\right)$ if $a_{i_{1} j_{1}}, a_{i_{1} j_{2}}$ and $a_{i_{2} j_{1}}$ are in $A-C\left(1 \leqq i_{1}, j_{1}, i_{2}, j_{2} \leqq n\right)$, then $a_{i_{2} j_{2}}$ are also in $A-C$,

$\left(\mathrm{A}_{2}\right)$ for any $a_{i j} \in C$ there exists some $a_{k \ell} \in C$ such that $(i, j) \leftrightarrow(k, \ell)$,

$\left(\mathrm{A}_{3}\right)$ if $a_{i_{\sigma} j_{\sigma}}, a_{k_{\sigma} \ell_{\sigma}} \in C(1 \leqq \sigma \leqq s)$ satisfy the conditions

$$
\left(i_{1}, j_{1}\right) \leftrightarrow\left(k_{1}, \ell_{1}\right),\left(i_{2}, j_{2}\right) \leftrightarrow\left(k_{2}, \ell_{2}\right), \cdots,\left(i_{s}, j_{s}\right) \leftrightarrow\left(k_{s}, \ell_{s}\right),
$$

then $\left\{i_{1}, i_{2}, \cdots, i_{s}\right\}=\left\{k_{1}, k_{2}, \cdots, k_{s}\right\}$ occurs when and only when $\left\{j_{1}, j_{2}, \cdots, j_{s}\right\}$ 
$=\left\{\ell_{1}, \ell_{2}, \cdots, \ell_{s}\right\}$, where some indices may appear repeatedly in $\left\{i_{1}, \cdots, i_{s}\right\}$ etc. and the equalities mean in this case that they appear the same times in both sides.

In this situation, we give

MAIN Lemma. By changing indices $i$ and $j$ of $a_{i j}$ 's individually, it holds that

(i) there is a partition of indices

$$
\{1,2, \cdots, n\}=\left\{1,2, \cdots, m_{1}\right\} \cup\left\{m_{1}+1, \cdots, m_{2}\right\} \cup \cdots \cup\left\{m_{t-1}+1, \cdots, n\right\}
$$

such that $a_{i j} \notin C$ if and only if $i$ and $j$ are in the same class $\left\{m_{\tau-1}+1, \cdots, m_{\tau}\right\}$ for some $\tau(1 \leqq \tau \leqq t)$, where $m_{0}:=0, m_{t}:=n$ and $t \geqq 2$, (ii) for any $a_{i j}, a_{k \ell}$ in $C,(i, j) \leftrightarrow(k, \ell)$ if and only if $i=\ell$ and $j=k$.

For the proof, we need some preparations.

LEMMA 2.1. For any $i(1 \leqq i \leqq n)$, there exist some $j_{1}$ and $j_{2}$ such that $a_{i j_{1}}$ and $a_{j_{2} i}$ are in $A-C$.

Proof. Assume that $a_{i j} \in C$ for any $j(1 \leqq j \leqq n)$. By the assumption $\left(\mathrm{A}_{2}\right)$, we can take some $k_{j}, \ell_{j}$ such that $(i, j) \leftrightarrow\left(k_{j}, \ell_{j}\right)$ for each $j$. Here, $j \neq \ell_{j}$. In fact, if not, $i \neq k_{j}$, which contradicts the assumption $\left(\mathrm{A}_{3}\right)$. And, $i \neq k_{j}$ by the same reason. Since $\{1,2, \cdots, i-1, i+1, \cdots, n\}$ cannot contain $n$ distinct elements, we have indices $j^{\prime}, j^{\prime \prime}$ such that $k_{j^{\prime}}=$ $k_{j^{\prime \prime}}$ and $j^{\prime} \neq j^{\prime \prime}$. Then, for the relations

$$
\left(i, j^{\prime}\right) \leftrightarrow\left(k_{j^{\prime}}, \ell_{j^{\prime}}\right),\left(k_{j^{\prime \prime}}, \ell_{j^{\prime \prime}}\right) \leftrightarrow\left(i, j^{\prime \prime}\right),
$$

$\left\{i, k_{j^{\prime \prime}}\right\}=\left\{k_{j^{\prime}}, i\right\}$ but $\left\{j^{\prime}, \ell_{j^{\prime \prime}}\right\} \neq\left\{\ell_{j^{\prime}}, j^{\prime \prime}\right\}$. This contradicts the assumption $\left(\mathrm{A}_{3}\right)$. Thus, there exists some $j_{1}$ such that $a_{i j_{1}} \notin C$. The existence of $j_{2}$ with $a_{j_{2} i} \notin C$ is shown similarly.

We introduce here a provisional notation. For integers $k, l$ with $k \leqq \ell$, we denote by $[k, \ell]$ the set of all integers $i$ with $k \leqq i \leqq \ell$.

By a suitable change of indices, we may assume $a_{i 1} \notin C$ for any $i \in[1, m]$ and $a_{j_{1}} \in C$ for any $j \in[m+1, n]$, where $1 \leqq m \leqq n-1$ by Lemma 2.1. Then, as is easily seen by the assumption $\left(\mathrm{A}_{1}\right)$, if $a_{i_{0} k_{0}} \notin C$ for some $k_{0} \in[2, n]$ and $i_{0} \in[1, m]$, then $a_{i k_{0}} \notin C$ for any $i \in[1, m]$ and $a_{j k_{0}} \in C$ for any $j \in[m+1, n]$. By this reason, choosing indices suitably, we may assume that $a_{i j} \notin C$ if $i \in[1, m], j \in\left[1, m^{\prime}\right]$ and $a_{i j} \in C$ if $i \in$ 
$[m+1, n], j \in\left[1, m^{\prime}\right]$, or $i \in[1, m], j \in\left[m^{\prime}+1, n\right]$, where $1 \leqq m^{\prime} \leqq n-1$. Moreover, it may be assumed that

(2.2) there are indices $m_{1}, \cdots, m_{t-1}, m_{1}^{\prime}, \cdots, m_{t-1}^{\prime}$ with

$$
\begin{aligned}
& m=: m_{1}<m_{2}<\ldots<m_{t-1}<m_{t}:=n \\
& m^{\prime}=: m_{1}^{\prime}<m_{2}^{\prime}<\ldots<m_{t-1}^{\prime}<m_{t}^{\prime}:=n
\end{aligned}
$$

such that $a_{i j} \notin C$ if and only if $i \in\left[m_{\tau-1}+1, m_{\tau}\right]$ and $j \in\left[m_{\tau-1}^{\prime}+1, m_{\tau}^{\prime}\right]$ for some $\tau \in[1, t]$, where we put $m_{0}=m_{0}^{\prime}=0$.

Later, $m_{\tau}=m_{\tau}^{\prime}(1 \leqq \tau \leqq t)$ will be shown. We assume $m^{\prime} \leqq m$ for a while by exchanging the situations of indices $i$ and $j$ of $a_{i j}$ if necessary.

For each $j$ in $\left[m^{\prime}+1, n\right]$, we define an index $I$, as follows.

(2.3) If $(1, j) \leftrightarrow(i, \ell)$ for any $i \in[1, m]$ and $\ell \in\left[m^{\prime}+1, n\right]$, we put $I_{\jmath}=1$. Otherwise, choose indices $i_{1}, i_{2}, \cdots, i_{a}$ in $[1, m]$ and $\ell_{1}, \ell_{2}, \cdots, \ell_{a}$ in $\left[m^{\prime}+1, n\right]$ such that

$$
(1, j) \leftrightarrow\left(i_{1}, \ell_{1}\right),\left(i_{1}, j\right) \leftrightarrow\left(i_{2}, \ell_{2}\right), \ldots,\left(i_{a-1}, j\right) \leftrightarrow\left(i_{a}, \ell_{a}\right)
$$

and $\left(i_{a}, j\right) \leftrightarrow(i, \ell)$ for any $i \in[1, m], \ell \in\left[m^{\prime}+1, n\right]$. And, put $I_{j}:=i_{a}$.

These choices are certainly possible. Indeed, if we cannot choose the above $i_{a}$, then there are infinitely many $i_{\beta} \in[1, m], \ell_{\beta} \in\left[m^{\prime}+1, n\right]$ $(\beta=1,2, \cdots)$ such that $\left(i_{\beta}, j\right) \leftrightarrow\left(i_{\beta+1}, \ell_{\beta+1}\right)$. We have necessarily $i_{\beta}=i_{\beta^{\prime}}$, for some $\beta$, $\beta^{\prime}$ with $\beta+2 \leqq \beta^{\prime}$ and relations

$$
\left(i_{\beta}, j\right) \leftrightarrow\left(i_{\beta+1}, \ell_{\beta+1}\right),\left(i_{\beta+1}, j\right) \leftrightarrow\left(i_{\beta+2}, \ell_{\beta+2}\right), \cdots,\left(i_{\beta^{\prime}-1}, j\right) \leftrightarrow\left(i_{\beta^{\prime}}, \ell_{\beta^{\prime}}\right)
$$

This contradicts the assumption $\left(\mathrm{A}_{3}\right)$, because

$$
\left\{i_{\beta}, \cdots, i_{\beta^{\prime}-1}\right\}=\left\{i_{\beta+1}, \cdots, i_{\beta^{\prime}}\right\}
$$

but

$$
\{j, \cdots, j\} \neq\left\{\ell_{\beta+1}, \cdots, \ell_{\beta^{\prime}}\right\} .
$$

LEMMA 2.4. If there are indices $k_{0} \in[m+1, n], \ell_{0}, \ell_{0}^{\prime}$ in $[1, n]$ such that

$(*)_{1}$

$$
\left(I_{j}, j\right) \leftrightarrow\left(k_{0}, \ell_{0}\right),\left(k_{0}, \ell_{0}^{\prime}\right) \leftrightarrow\left(I_{j^{\prime}}, j^{\prime}\right)
$$

then $j=j^{\prime}$.

Proof. As in (2.3), we can take indices $i_{1}, \cdots, i_{a-1}, i_{1}^{\prime}, \cdots, i_{a^{\prime}-1}^{\prime}$ in $[1, m]$ and $\ell_{1}, \cdots, \ell_{a}, \ell_{1}^{\prime}, \cdots, \ell_{a^{\prime}}^{\prime}$ in $\left[m^{\prime}+1, n\right]$ such that 
$(*)_{2}$

$$
\begin{aligned}
& (1, j) \leftrightarrow\left(i_{1}, \ell_{1}\right),\left(i_{1}, j\right) \leftrightarrow\left(i_{2}, \ell_{2}\right), \cdots,\left(i_{a-1}, j\right) \leftrightarrow\left(I_{j}, \ell_{a}\right) \\
& \left(i_{1}^{\prime}, \ell_{1}^{\prime}\right) \leftrightarrow\left(1, j^{\prime}\right),\left(i_{2}^{\prime}, \ell_{2}^{\prime}\right) \leftrightarrow\left(i_{1}^{\prime}, j^{\prime}\right), \cdots,\left(I_{j^{\prime}}, \ell_{a^{\prime}}^{\prime}\right) \leftrightarrow\left(i_{a^{\prime}-1}^{\prime}, j^{\prime}\right) .
\end{aligned}
$$

For the relations $(*)_{1}$ and $(*)_{2}$, we see

$$
\begin{aligned}
& \left\{I_{j}, k_{0}, 1, i_{1}, \cdots, i_{a-1}, i_{1}^{\prime}, \cdots, i_{a^{\prime}-1}^{\prime}, I_{j^{\prime}}\right\} \\
& \quad=\left\{k_{0}, I_{j^{\prime}}, i_{1}, \cdots, i_{a-1}, I_{j}, 1, i_{1}^{\prime}, \cdots, i_{a^{\prime}-1}^{\prime}\right\} .
\end{aligned}
$$

So, by the assumption $\left(A_{3}\right)$

$$
\left\{j, \ell_{0}^{\prime}, j, \cdots, j, \ell_{1}^{\prime}, \cdots, \ell_{a^{\prime}}^{\prime}\right\}=\left\{\ell_{0}, j^{\prime}, \ell_{1}, \cdots, \ell_{a}, j^{\prime}, \cdots, j^{\prime}\right\} .
$$

This implies $j=j^{\prime}$ because $j \neq \ell_{0}, \ell_{1}, \cdots, \ell_{a}, j^{\prime}$.

LEMMA 2.5. For any $k \in[m+1, n]$ there is one and only one $j \in\left[m^{\prime}+1, n\right]$ such that $\left(I_{j}, j\right) \leftrightarrow(k, \ell)$ for some $\ell \in[1, n]$.

Proof. The uniqueness of the desired index is a result of Lemma 2.4. On the other hand, by the assumption $\left(\mathrm{A}_{2}\right)$, there are indices $k_{m^{\prime}+1}, \cdots, k_{n}, \ell_{m^{\prime}+1}, \cdots, \ell_{n}$ such that

$$
\left(I_{m^{\prime}+1}, m^{\prime}+1\right) \leftrightarrow\left(k_{m^{\prime}+1}, \ell_{m^{\prime}+1}\right), \cdots,\left(I_{n}, n\right) \leftrightarrow\left(k_{n}, \ell_{n}\right),
$$

where $m+1 \leqq k_{m^{\prime}+1}, \cdots, k_{n} \leqq n$ by the property (2.3) of $I_{j}$ 's. Then, $k_{m+1}, \cdots, k_{n}$ are distinct with each other because of Lemma 2.4. Therefore,

$$
n-m^{\prime} \leqq n-m
$$

and so $m \leqq m^{\prime}$. Since $m^{\prime} \leqq m$ is assumed, we have $m=m^{\prime}$ and $\left\{k_{m+1}, \cdots, k_{n}\right\}=\{m+1, \cdots, n\}$. The index $j$ with $k_{j}=k$ is the desired one.

LEMMA 2.6. $\quad m_{\tau}=m_{\tau}^{\prime}(1 \leqq \tau \leqq t)$ for the numbers defined as in (2.2).

Proof. As in the proof of Lemma 2.5, we have $m\left(=m_{1}\right)=m^{\prime}\left(=m_{1}^{\prime}\right)$. The same arguments are available for the other $\tau$. So, we obtain Lemma 2.6.

LEMMA 2.7. For any $i \in[m+1, n]$ and $j \in[1, m]$ there exist some $k \in[1, m]$ and $\ell \in[m+1, n]$ such that $(i, j) \leftrightarrow(k, \ell)$.

Proof. Assume the contrary. According to the assumtion $\left(\mathrm{A}_{2}\right)$, we choose indices $k_{0}, \ell_{0} \in[1, n]$ such that

$$
(i, j) \leftrightarrow\left(k_{0}, \ell_{0}\right)
$$


By the assumption, $m+1 \leqq k_{0} \leqq n$. On the other hand, there are indices $j_{0}, j_{0}^{\prime}$ in $[m+1, n]$ and $\ell_{0}^{\prime}, \ell_{0}^{\prime \prime}$ in $[1, n]$ such that

$(\#)_{2}$

$$
\left(I_{j_{0}}, j_{0}\right) \leftrightarrow\left(i, \ell_{0}^{\prime}\right),\left(k_{0}, \ell_{0}^{\prime \prime}\right) \leftrightarrow\left(I_{j_{0}}, j_{0}^{\prime}\right)
$$

because of Lemma 2.5. Moreover, by the property (2.3) of $I_{j}$ 's, we have

$$
\begin{aligned}
& \left(1, j_{0}\right) \leftrightarrow\left(i_{1}, \ell_{1}\right),\left(i_{1}, j_{0}\right) \leftrightarrow\left(i_{2}, \ell_{2}\right), \cdots,\left(i_{a-1}, j_{0}\right) \leftrightarrow\left(I_{j_{0}}, \ell_{a}\right) \\
& \left(i_{1}^{\prime}, \ell_{1}^{\prime}\right) \leftrightarrow\left(1, j_{0}^{\prime}\right),\left(i_{2}^{\prime}, \ell_{2}^{\prime}\right) \leftrightarrow\left(i_{1}^{\prime}, j_{0}^{\prime}\right), \cdots,\left(I_{j_{0}^{\prime}}, \ell_{a^{\prime}}^{\prime}\right) \leftrightarrow\left(i_{a^{\prime}-1}^{\prime}, j_{0}^{\prime}\right)
\end{aligned}
$$

for some $i_{1}, \cdots, i_{a-1}, i_{1}^{\prime}, \cdots, i_{a^{\prime}-1}^{\prime} \in[1, m]$ and $\ell_{1}, \cdots, \ell_{a}, \ell_{1}^{\prime}, \cdots, \ell_{a^{\prime}}^{\prime}$ in $[m+1, n]$. Observe the indices of the relations $(\#)_{1},(\#)_{2}$ and $(\#)_{3}$. It is easily seen that they contradict the assumption $\left(A_{3}\right)$. Thus, we have Lemma 2.7.

LEMMA 2.8. By a suitable change of indices $i$ 's of $a_{i j}$ among $1,2, \cdots, m$, there is some index $\ell_{i j}$ for each $i \in[m+1, n]$ and $j \in[1, m]$ such that $(i, j) \leftrightarrow\left(j, \ell_{i j}\right)$, where $m+1 \leqq \ell_{i j} \leqq n$.

Proof. We take $k_{1}, \cdots, k_{m}$ in $[1, m]$ and $\ell_{1}, \cdots, \ell_{m}$ in $[m+1, n]$ such that

$$
(m+1,1) \leftrightarrow\left(k_{1}, \ell_{1}\right), \cdots,(m+1, m) \leftrightarrow\left(k_{m}, \ell_{m}\right)
$$

by the use of Lemma 2.7. As is easily seen by the assumption $\left(A_{0}\right)$ and $\left(\mathrm{A}_{3}\right)$, we have $\left\{k_{1}, \cdots, k_{m}\right\}=\{1, \cdots, m\}$. By a change of indices, we may assume that $k_{1}=1, \cdots, k_{m}=m$. For any $i \in[m+1, n]$, we choose $k_{1}^{\prime}, \cdots, k_{m}^{\prime}$ in $[1, m]$ and $\ell_{1}^{\prime}, \cdots \ell_{m}^{\prime}$ in $[m+1, n]$ so that

$$
(i, 1) \leftrightarrow\left(k_{1}^{\prime}, \ell_{1}^{\prime}\right), \cdots,(i, m) \leftrightarrow\left(k_{m}^{\prime}, \ell_{m}^{\prime}\right) .
$$

By the same reason as the above, $\left\{k_{1}^{\prime}, \cdots, k_{m}^{\prime}\right\}=\{1,2, \cdots, m\}$. Assume that $k_{j}^{\prime} \neq j$ for some $j$ and take the index $j^{\prime}$ with $k_{j^{\prime}}^{\prime}=j$. We observe the relations

$$
(i, j) \leftrightarrow\left(k_{j}^{\prime}, \ell_{j}^{\prime}\right),\left(k_{j}^{\prime}, \ell_{k_{j}^{\prime}}\right) \leftrightarrow\left(m+1, k_{j}^{\prime}\right),(m+1, j) \leftrightarrow\left(j, \ell_{j}\right),\left(j, \ell_{j^{\prime}}^{\prime}\right) \leftrightarrow\left(i, j^{\prime}\right) .
$$

As is easily seen by the facts $j \neq \ell_{j}^{\prime}, k_{j}^{\prime}, \ell_{j}, j^{\prime}$, this contradicts the assumption $\left(\mathrm{A}_{3}\right)$. Therefore, $k_{j}^{\prime}=j$ for any $j$ and we have Lemma 2.8.

LEMMA 2.9. After a suitable change of indices $j$ 's of $a_{i j}$ among $m+1, \cdots, n$, it holds that $(i, j) \leftrightarrow(j, i)$ for any $j \in[m+1, n]$ and $j \in[1, m]$.

Proof. As a consequence of Lemma 2.8, we may assume that 


$$
(m+1,1) \leftrightarrow\left(1, \ell_{m+1}\right), \cdots,(n, 1) \leftrightarrow\left(1, \ell_{n}\right),
$$

where $\left\{\ell_{m+1}, \cdots, \ell_{n}\right\}=\{m+1, \cdots, n\}$ by the assumption $\left(\mathrm{A}_{3}\right)$. Changing indices if necessary, we have $(\ell, 1) \leftrightarrow(1, \ell)$ for any $\ell \in[m+1, n]$. Assume that for some $i_{0} \in[1, m]$ and $j_{0} \in[m+1, n]\left(i_{0}, j_{0}\right) \leftrightarrow\left(j_{0}, i_{0}\right)$. Then, by Lemma 2.8, there is some $\ell_{0} \in[m+1, n]\left(j_{0}, i_{0}\right) \leftrightarrow\left(i_{0}, \ell_{0}\right)$ such that $\ell_{0} \neq j_{0}$. If we choose $k_{m+1}, \cdots, k_{n}$ in $[m+1, n]$ such that $\left(j, i_{0}\right) \leftrightarrow\left(i_{0}, k_{j}\right)$ for each $j \in[m+1, n]$, it is easily seen that $\left\{k_{m+1}, \cdots, k_{n}\right\}=\{m+1, \cdots, n\}$. Therefore, there are an index $k_{0}$ such that $\left(k_{0}, i_{0}\right) \leftrightarrow\left(i_{0}, j_{0}\right)$, where $k_{0} \neq j_{0}$ by the assumption. We observe the relations

$$
\left(i_{0}, \ell_{0}\right) \leftrightarrow\left(j_{0}, i_{0}\right),\left(k_{0}, i_{0}\right) \leftrightarrow\left(i_{0}, j_{0}\right),\left(1, k_{0}\right) \leftrightarrow\left(k_{0}, 1\right),\left(j_{0}, 1\right) \leftrightarrow\left(1, j_{0}\right) .
$$

Obviously, these indices do not satisfy the assumption $\left(\mathrm{A}_{3}\right)$. Thus, we get Lemma 2.9.

Proof of Main Lemma. By Lemma 2.9, we may assume that $(i, j)$ $\leftrightarrow(j, i)$ for any $i \in[m+1, n]$ and $j \in[1, m]$. The conclusion (i) of Main Lemma is a direct result of Lemma 2.6 because Lemma 2.6 is available for the above choice of indices. We shall prove the conclusion (ii). There are indices $k, \ell$ with $(i, j) \leftrightarrow(k, \ell)$ for any $i, j$ with $a_{i j} \in C$ by the assumption $\left(\mathrm{A}_{2}\right)$. So, we have only to show that $k=j$ and $\ell=i$ whenever $(i, j) \leftrightarrow(k, \ell)$. By virtue of the assumption $\left(\mathrm{A}_{0}\right)$, it suffices to study the following three cases.

$\left.1^{\circ}\right) \quad m+1 \leqq i \leqq n, 1 \leqq j \leqq m, 1 \leqq k \leqq m$ and $m+1 \leqq \ell \leqq n$.

$\left.2^{\circ}\right) \quad m+1 \leqq i \leqq n, 1 \leqq j \leqq m, m+1 \leqq k \leqq n$ and $1 \leqq \ell \leqq n$.

$\left.3^{\circ}\right) m+1 \leqq i, j, k, \ell \leqq n$.

Observe the relations

$$
(i, j) \leftrightarrow(k, \ell),(j, i) \leftrightarrow(i, j),(k, \ell) \leftrightarrow(\ell, k),(\ell, j) \leftrightarrow(j, \ell)
$$

for the case $1^{\circ}$ ) and

$$
(i, j) \leftrightarrow(k, \ell),(1, i) \leftrightarrow(i, 1),(k, 1) \leftrightarrow(1, k),
$$

for the cases $2^{\circ}$ ) and $3^{\circ}$ ) respectively. In any case, indices in the relations do not satisfy the assumption $\left(\mathrm{A}_{3}\right)$ except the case $(k, \ell)=(j, i)$. Thus, Main Lemma is completely proved.

\section{§3. A result from the value distribution theory}

We shall introduce some definitions and notations. For a domain 
$D$ in the complex plane $C$, a divisor $\nu(z)$ on $D$ is defined as an integervalued function on $D$ such that $\{z \in D ; \nu(z) \neq 0\}$ has no accumulation point in $D$. Let us take a divisor $\nu$ on $\{z \in C ;|z|<R\}(0<R \leqq+\infty)$ with $\nu(0)=0$. We put

$$
\begin{aligned}
& n(r, \nu):=\sum_{|z| \leqq r} \nu(z) \\
& N(r, \nu):=\int_{0}^{r} \frac{n(t, \nu)}{t} d t=\sum_{|z| \leqq r} \nu(z) \log \frac{r}{|z|},
\end{aligned}
$$

where $0 \leqq r \leqq R$.

Let $f$ be a non-constant meromorphic function on $C$. We define $\nu_{f}(a)=n,=0$ and $=-m$ if $f(z)$ has a zero of order $n$ at $z=a$, if $f(a) \neq 0$ and if $f(z)$ has a pole of order $m$ at $z=a$, respectively. And, put $N(r, f)=N\left(r, \nu_{f}\right)$. Then, the well-known Jensen's formula is given as follows.

(3.1) If $f(0) \neq 0, \infty$, then

$$
\frac{1}{2 \pi} \int_{0}^{2 \pi} \log \left|f\left(r e^{i \theta}\right)\right| d \theta=N(r, f)+\log |f(0)| \quad(r>0) .
$$

Now, let us take a holomorphic map $f$ of $C$ into $P^{N}(C)$. For an arbitrarily fixed homogeneous coordinates $w_{1}: \ldots: w_{N+1}$, we can take holomorphic functions $f_{1}, \cdots, f_{N+1}$ such that $f=f_{1}: \cdots: f_{N+1}$ and $f_{i}$ $(1 \leqq i \leqq N+1)$ have no common zeros. In the following, we shall call such a representation of $f$ a reduced representation. For a reduced representation $f=f_{1}: f_{2}: \cdots: f_{N+1}$, we put

$$
u(z):=\max _{1 \leqq i \leqq N+1} \log \left|f_{i}(z)\right|
$$

and, following H. Cartan [2], define the characteristic function of $f$ as

$$
T(r, f)=\frac{1}{2 \pi} \int_{0}^{2 \pi} u\left(r e^{i \theta}\right) d \theta-u(0)
$$

which is determined independently of any choice of a reduced representation of $f$.

Assume that $f$ is non-degenerate, i.e., $f(C)$ is not contained in any hyperplane of $P^{N}(C)$. For a hyperplane

$$
H: a^{1} w_{1}+a^{2} w_{2}+\cdots+a^{N+1} w_{N+1}=0
$$


and a reduced representation $f=f_{1}: f_{2}: \cdots: f_{N+1}$, we consider a holomorphic function

$$
F:=a^{1} f_{1}+a^{2} f_{2}+\cdots+a^{N+1} f_{N+1}
$$

and define $\nu(f, H):=\nu_{F}$.

Definition 3.2. For a positive integer $p$, we define

$$
\begin{aligned}
& N_{p}(r, f, H):=N(r, \min (p, \nu(f, H))) \\
& N(r, f, H):=N(r, \nu(f, H)) .
\end{aligned}
$$

We can conclude from (3.1)

$$
N_{p}(r, f, H) \leqq N(r, f, H) \leqq T(r, f)+K,
$$

where $K$ is a constant not depending on $r$.

We recall here the second fundamental theorem in the value distribution theory given by H. Cartan in [2], which is essentially used in the followings.

THEOREM 3.4. Let $f$ be a non-degenerate holomorphic map of $C$ into $P^{N}(C)$ and $H_{i}(1 \leqq i \leqq q)$ be hyperplanes in general position with $f(0) \notin \cup_{i} H_{i}$. Then,

$$
(q-N-1) T(r, f) \leqq \sum_{1 \leqq i \leqq q} N_{N}\left(r, f, H_{i}\right)+S(r),
$$

where

$$
S(r)=O(\log r)+O(\log T(r, f))
$$

and "\|" means that this holds outside an open set $E$ in $\boldsymbol{R}$ such that $\int_{E} \frac{d t}{t}<+\infty$

Remark. In Theorem 3.4, if $f$ is rational, i.e., represented as $f=f_{1}: f_{2}: \cdots: f_{N+1}$ with polynomials $f_{i}$, then $S(r)=O(1)$.

Now, let us consider two non-degenerate holomorphic maps $f, g$ of $C$ into $P^{N}(C)$ and $N+2$ hyperplanes $H_{1}, \cdots, H_{N+2}$ in general position. We assume that

$$
\nu\left(f, H_{i}\right)=\nu\left(g, H_{i}\right)=0
$$

i.e., $f(C) \cap H_{i}=g(C) \cap H_{i}=\phi$ for $i=1,2, \cdots, N+1$ and

$$
\min \left(\nu\left(f, H_{N+2}\right), N\right)=\min \left(\nu\left(g, H_{N+2}\right), N\right) .
$$


We choose homogeneous coordinates $w_{1}: w_{2}: \cdots: w_{N+1}$ on $P^{N}(C)$ such that $H_{i}$ are represented as

$$
\begin{aligned}
& H_{i}: w_{i}=0 \quad 1 \leqq i \leqq N+1, \\
& H_{N+2}: w_{1}+w_{2}+\cdots+w_{N+1}=0
\end{aligned}
$$

In this situation, we can prove the following

PRoposition 3.7. Take reduced representations $f=f_{1}: f_{2}: \cdots: f_{N+1}$ and $g=g_{1}: g_{2}: \cdots: g_{N+1}$. Then there exists some constants $c_{1}, c_{2}, \cdots$, $c_{N+1}, d_{1}, d_{2}, \cdots, d_{N+1}$ such that $c_{i}-d_{j} \neq 0$ for some $i, j$ and

$$
\sum_{1 \leqq i, j \leqq N+1}\left(c_{i}-d_{j}\right) f_{i} g_{j}=0 \text {. }
$$

To prove this, we need some preparations. For brevity, we denote $H_{N+2}$ by $H$ and define

$$
\begin{aligned}
& N^{\prime}(r, f):=N(r, \nu(f, H)-\min (\nu(f, H), \nu(g, H))) \\
& N^{\prime}(r, g):=N(r, \nu(g, H)-\min (\nu(f, H), \nu(g, H)))
\end{aligned}
$$

for each positive number $r$.

LEMMA 3.9. It holds that

$$
N^{\prime}(r, f)+N^{\prime}(r, g) \leqq N(r, f, H)-N_{N}(r, f, H)+N(r, g, H)-N_{N}(r, g, H) \text {. }
$$

Proof. According to the assumption (3.6), we see easily

$$
\begin{aligned}
(\nu(f, H)-\min (\nu(f, H), \nu(g, H)))+(\nu(g, H)-\min (\nu(f, H), \nu(g, H))) \\
\quad=|\nu(f, H)-\nu(g, H)| \\
\quad \leqq|\nu(f, H)-\min (\nu(f, H), N)|+|\nu(g, H)-\min (\nu(f, H), N)| \\
\quad=(\nu(f, H)-\min (\nu(f, H), N))+(\nu(g, H)-\min (\nu(g, H), N)) .
\end{aligned}
$$

By linearlity and monotonicity of integrals, we can conclude Lemma 3.9.

LEMMA 3.10. It holds that

$$
N^{\prime}(r, f)+N^{\prime}(r, g)=O(\log r)+O(\log (T(r, f)+T(r, g))) \| .
$$

Here, if $f$ and $g$ are both rational, the right hand side is replaced by $O(1)$.

Proof. Since $N_{N}\left(r, f, H_{i}\right)=N_{N}\left(r, g, H_{i}\right)=0(1 \leqq i \leqq N+1)$ by the assumption (3.5), Theorem 3.4 implies that 


$$
\begin{aligned}
& T(r, f)-N_{N}(r, f, H)=O(\log r)+O(\log T(r, f)) \|, \\
& T(r, g)-N_{N}(r, g, H)=O(\log r)+O(\log T(r, g)) \quad \| .
\end{aligned}
$$

Therefore, by (3.3), we see

$$
\begin{aligned}
& N(r, f, H)-N_{N}(r, f, H)=O(\log r)+O(\log T(r, f)) \quad \|, \\
& N(r, g, H)-N_{N}(r, f, H)=O(\log r)+O(\log T(r, g)) \quad \| .
\end{aligned}
$$

By virtue of Lemma 3.9, we can conclude

$$
\begin{aligned}
& N^{\prime}(r, f)+N^{\prime}(r, g) \\
& \quad \leqq O(\log r)+O(\log T(t, f) T(r, g)) \\
& \quad \leqq O(\log r)+O(\log (T(r, f)+T(r, g)))
\end{aligned}
$$

The latter half of Lemma 3.10 is due to Remark to Theorem 3.4.

Proof of Proposition 3.7. We take a holomorphic function $h$ on $C$ such that $\nu_{h}=\min (\nu(f, H), \nu(g, H))$. And, we consider a holomorphic map $\Phi$ of $C$ into $P^{2 N}(C)$ defined as

$$
\Phi=f_{1} \tilde{g}: f_{2} \tilde{g}: \cdots: f_{N+1} \tilde{g}:-g_{1} \tilde{f}: \cdots:-g_{N} \tilde{f},
$$

for some fixed homogeneous coordinates on $P^{2 N}(C)$, where $\tilde{f}:=f_{1}+\cdots$ $+f_{N+1} / h$ and $\tilde{g}:=g_{1}+\cdots+g_{N+1} / h$. Since $f_{i}$ and $g_{i}(1 \leqq i \leqq N+1)$ have no zeros and $\tilde{f}$ and $\tilde{g}$ have no common zeros, (3.11) is a reduced representation of $\Phi$. For the proof of Proposition 3.7, we have only to show that $\Phi$ is degenerate. In fact, in this case, there exist some constants $c_{1}, \cdots, c_{N+1}, d_{1}, d_{2}, \cdots, d_{N}$, at least one of which is not zero, such that

$$
\sum_{1 \leq i \leq N+1} c_{i} f_{i}\left(g_{1}+\cdots+g_{N+1}\right)-\sum_{1 \leqq j \leqq N} d_{j} g_{j}\left(f_{1}+\cdots+f_{N+1}\right)=0 .
$$

Here, at least one of $c_{i}$ 's is not zero, because $g$ is non-degenerate. Putting $d_{N+1}=0$, we have the desired relation (3.8).

Now, let us assume that $\Phi$ is non-degenerate. We denote by $u_{1}$ : $u_{2}: \cdots: u_{2 N+1}$ the above fixed homogeneous coordinates on $P^{2 N}(C)$ and consider $2 N+2$ hyperplanes

$$
\begin{aligned}
& \tilde{H}_{i}: u_{i}=0 \quad 1 \leqq i \leqq 2 N+1, \\
& \tilde{H}_{2 N+2}: u_{1}+u_{2}+\cdots+u_{2 N+1}=0
\end{aligned}
$$

in $P^{2 N}(C)$, which are located in general position. Then, 


$$
\begin{aligned}
\nu\left(\Phi, H_{i}\right) & =\nu_{\tilde{g}}=\nu(g, H)-\min (\nu(f, H), \nu(g, H)) & & \text { if } 1 \leqq i \leqq N+1, \\
& =\nu_{\tilde{f}}=\nu(f, H)-\min (\nu(f, H), \nu(g, H)) & & \text { if } N+2 \leqq i \leqq 2 N+1 .
\end{aligned}
$$

Moreover, since $\sum_{1 \leqq i \leqq N+1}\left(\tilde{g} f_{i}-\tilde{f} g_{i}\right)=0$,

$$
\nu\left(\Phi, H_{2 N+2}\right)=\nu_{\tilde{f} g_{N+1}}=\nu_{\tilde{f}} .
$$

We apply here Theorem 3.4 to a holomorphic map $\Phi$ of $C$ into $P^{2 N}(C)$ and hyperplanes $H_{1}, \cdots, H_{2 N+2}$. We have

$$
\begin{aligned}
T(r, \Phi) & \leqq \sum_{1 \leqq j \leqq 2 N+2} N_{2 N}\left(r, \Phi, \tilde{H}_{j}\right)+O(\log r T(r, \Phi)) \\
& \leqq(N+1)\left(N^{\prime}(r, f)+N^{\prime}(r, g)\right)+O(\log r T(r, \Phi))
\end{aligned}
$$

Put

$$
\begin{aligned}
u_{\Phi} & :=\max \left(\log \left|f_{1} \tilde{g}\right|, \cdots, \log \left|f_{N+1} \tilde{g}\right|, \log \left|g_{1} \tilde{f}\right|, \cdots, \log \left|g_{N} \tilde{f}\right|\right) \\
u_{f} & :=\max \left(\log \left|f_{1}\right|, \log \left|f_{2}\right|, \cdots, \log \left|f_{N+1}\right|\right) \\
u_{g} & :=\max \left(\log \left|g_{1}\right|, \cdots, \log \left|g_{N}\right|\right)=\max \left(\log \left|g_{1}\right|, \cdots, \log \left|g_{N+1}\right|\right),
\end{aligned}
$$

where we used a reduced representation of $g$ with $g_{N+1} \equiv 1$. Then,

$$
u_{\Phi}(z) \geqq\left\{\begin{array}{l}
u_{f}(z)+\log |\tilde{g}(z)| \\
u_{g}(z)+\log |\tilde{f}(z)| .
\end{array}\right.
$$

Taking the mean value of each term on $\{z \in C ;|z|=r\}$, we obtain by (3.1)

$$
\begin{aligned}
& T(r, \Phi)+u_{\Phi}(0) \\
& \geqq\left\{\begin{array}{l}
T(r, f)+u_{f}(0)+N(r, \tilde{g})+\log |\tilde{g}(0)| \\
T(r, g)+u_{g}(0)+N(r, \tilde{f})+\log |\tilde{f}(0)|
\end{array}\right.
\end{aligned}
$$

Here, $N(r, \tilde{g})=N^{\prime}(r, f)$ and $N(r, \tilde{f})=N^{\prime}(r, g)$. So, by (3.12),

$$
\begin{gathered}
T(r, f)+T(r, g) \leqq 2 T(r, \Phi)-N^{\prime}(r, f)-N^{\prime}(r, g)+O(1) \\
\leqq(2 N+1)\left(N^{\prime}(r, f)+N^{\prime}(r, g)\right)+O(\log r T(r, \Phi)) \quad \| .
\end{gathered}
$$

On the other hand, since $\max \left(\left|f_{1} \tilde{g}\right|, \cdots,\left|f_{N+1} \tilde{g}\right|,\left|g_{1} \tilde{f}\right|, \cdots,\left|g_{N} \tilde{f}\right|\right) \leqq(N+1)\left(\max _{i}\left|g_{i}\right|\right) \times\left(\max _{j}\left|f_{\jmath}\right|\right) /|h|$, we have

$$
u_{\Phi}(z) \leqq u_{f}(z)+u_{\theta}(z)-\log |h|+\log (N+1)
$$

and by (3.1) 


$$
\frac{1}{2 \pi} \int_{0}^{2 \pi} \log \left|\mathrm{h}\left(r e^{i \theta}\right)\right| d \theta=N(r, h)+\log |h(0)|
$$

Therefore,

$$
\begin{aligned}
T(r, \Phi) & \leqq T(r, f)+T(r, g)-N(r, h)+O(1) \\
& \leqq T(r, f)+T(r, g)+O(1) .
\end{aligned}
$$

$\mathrm{By}$ (3.13) and Lemma 3.10, we can conclude

$$
T(r, f)+T(r, g) \leqq O(\log r)+O(\log (T(r, f)+T(r, g))) \quad \| \cdot
$$

If $f$ or $g$ is transcendental, then

$$
\lim _{r \rightarrow \infty} \frac{\log r}{T(r, f)+T(r, g)}=0 .
$$

Factoring each term of the above inequality by $T(r, f)+T(r, g)$ and tending $r$ to the infinity, we have an absurd inequality. In the case that $f$ and $g$ are both rational, the remaining terms of the obtained inequalities in the above arguments can be replaced by $O(1)$. We have a contradiction in this case too. Therefore, $\Phi$ is degenerate and hence Proposition 3.7 is completely proved.

\section{§4. The Proofs of Theorems I and II}

We shall prove first Theorem II stated in $\S 1$ for the case $n=1$. Let $f, g$ be algebraically non-degenerate holomorphic maps of $C$ into $P^{N}(C)$ such that there are hyperplanes $H_{i}(1 \leqq i \leqq N+2)$ in general position satisfying the condition $\nu\left(f, H_{i}\right)=\nu\left(g, H_{i}\right)=0(1 \leqq i \leqq N+1)$ and

$$
\min \left(\nu\left(f, H_{N+2}\right), N\right)=\min \left(\nu\left(g, H_{N+2}\right), N\right) .
$$

As was shown in $\S 3$, if we choose homogeneous coordinates such that

$$
\begin{aligned}
& H_{i}: w_{i}=0 \quad 1 \leqq i \leqq N+1 \\
& H_{N+2}: w_{1}+\cdots+w_{N+1}=0
\end{aligned}
$$

and reduced representations $f=f_{1}: f_{2}: \cdots: f_{N+1}, g=g_{1}: g_{2}: \cdots: g_{N+1}$, we have the relation (3.8) for some constants $c_{i}$ and $d_{j}$, where $f_{i}$ and $g_{j}$ have no zeros.

We put $a_{i j}=f_{i} g_{j}$ and consider the set

$$
A:=\left\{a_{i j} ; 1 \leqq i, j \leqq N+1\right\} .
$$


And, we define subsets $C$ of $A$ and $\Gamma$ of $C \times C$ as

$$
\begin{aligned}
& C:=\left\{a_{i j} \in A ; c_{i}-d_{j} \neq 0 \text { for constants } c_{i}, d_{j} \text { as in }(3.8)\right\}, \\
& \Gamma:=\left\{\left(a_{i j}, a_{k \ell}\right) ; a_{i j} / a_{k \ell} \text { is of constant and }(i, j) \neq(k, \ell)\right\}
\end{aligned}
$$

respectively. For these sets, we shall show that the assumption $\left(A_{0}\right)$ $\sim\left(\mathrm{A}_{3}\right)$ in $\S 2$ are all satisfied. The assumption $\left(\mathrm{A}_{0}\right)$ is obviously valid. If $c_{i_{1}}-d_{j_{1}}=0, c_{i_{2}}-d_{j_{2}}=0$ and $c_{i_{1}}-d_{j_{2}}=0\left(1 \leqq i_{1}, i_{2}, j_{1}, j_{2} \leqq N+1\right)$, then

$$
c_{i_{2}}-d_{j_{2}}=\left(c_{i_{2}}-d_{j_{1}}\right)+\left(d_{j_{1}}-c_{i_{1}}\right)+\left(c_{i_{1}}-d_{f_{2}}\right)=0,
$$

whence $\left(A_{1}\right)$ is satisfied.

The assumption $\left(\mathrm{A}_{2}\right)$ can be easily seen by the relation (3.8) and the following classical theorem of E. Borel,

THEOREM 4.2 ([1]). Let $h_{1}, h_{2}, \cdots, h_{p}$ be nowhere vanishing holomorphic functions on $C$ satisfying the relation

$$
h_{1}+h_{2}+\cdots+h_{p}=0 \text {. }
$$

Then, there is a partition of the set of indices $I:=\{1,2, \cdots, p\}$ into the disjoint union of subsets

$$
I=I_{1} \cup \cdots \cup I_{k}
$$

such that for any $i, j \in I_{*} h_{i} / h_{j} \equiv$ const. and

$$
\sum_{i \in I_{\kappa}} h_{i} \equiv 0 \quad(1 \leqq \kappa \leqq k)
$$

Particularly, for any $i=1, \cdots, p$, there is some $j$ such that $i \neq j$ and $h_{i} / h_{j} \equiv$ const.

To verify the assumption $\left(\mathrm{A}_{3}\right)$, we take $a_{i_{\sigma} f_{\sigma}}$ and $a_{k_{\sigma} \ell_{\sigma}}(1 \leqq \sigma \leqq s)$ in $C$ satisfying the condition

$$
\left(i_{1}, j_{1}\right) \leftrightarrow\left(k_{1}, \ell_{1}\right),\left(i_{2}, j_{2}\right) \leftrightarrow\left(k_{2}, \ell_{2}\right), \ldots,\left(i_{s}, j_{s}\right) \leftrightarrow\left(k_{s}, \ell_{s}\right),
$$

namely, $f_{i_{\sigma}} g_{j_{\sigma}} / f_{k_{\sigma}} g_{\ell_{\sigma}} \equiv$ const. $(1 \leqq \sigma \leqq s)$. This implies that

$$
f_{i_{1}} f_{i_{2}} \cdots f_{i_{s}} g_{j_{1}} g_{j_{2}} \cdots g_{j_{s}}=c f_{k_{1}} f_{k_{2}} \cdots f_{k_{s}} g_{\ell_{1}} g_{\ell_{2}} \cdots g_{\ell_{s}}
$$

for some constant $c$. If $\left\{i_{1}, \cdots, i_{s}\right\}=\left\{k_{1}, \cdots, k_{s}\right\}$, we have a relation

$$
g_{j_{1}} g_{j_{2}} \cdots g_{j_{s}}=c g_{\ell_{1}} g_{\ell_{2}} \cdots g_{\ell_{s}} \text {. }
$$

On the other hand, there is no algebraic relation among $g_{1}, \ldots, g_{N+1}$ 
because $g$ is assumed to be algebraically non-degenerate. We can conclude $\left\{j_{1}, j_{2}, \cdots, j_{s}\right\}=\left\{\ell_{1}, \ell_{2}, \cdots, \ell_{s}\right\}$. Similarly, $\left\{j_{1}, \cdots, j_{s}\right\}=\left\{\ell_{1}, \cdots, \ell_{s}\right\}$ implies $\left\{i_{1}, \cdots, i_{s}\right\}=\left\{k_{1}, \cdots, k_{s}\right\}$. This shows that the assumption $\left(\mathrm{A}_{3}\right)$ is also satisfied.

By virtue of Main Lemma, we can conclude that, after a suitable change of indices $i$ and $j$ of $f_{i}$ and $g_{j}$ individually,

$$
f_{i} g_{j} / f_{k} g_{\ell} \equiv \text { const. }
$$

if and only if $(i, j)=(\ell, k)$ for any $(i, j)$ and $(k, \ell)$ with $c_{i}-d_{j} \neq 0$ and $c_{k}-d_{\ell} \neq 0$. Moreover, by the relation (3.8) and Theorem 4.2, we have

$$
f_{i} g_{j}-f_{j} g_{i} \equiv 0
$$

for any $i, j$ with $a_{i j} \in C$. In particular, as a result of (i) of Main Lemma,

$$
f_{i} g_{j}=f_{j} g_{i}
$$

if $m+1 \leqq i \leqq N+1,1 \leqq j \leqq m$ or $\quad 1 \leqq i \leqq m, m+1 \leqq j \leqq N+1$.

Easily we see

$$
\frac{f_{1}}{g_{1}}=\frac{f_{2}}{g_{2}}=\cdots=\frac{f_{N+1}}{g_{N+1}}
$$

Going back to the original indices, this shows that there is a permutation $\pi=\left(\begin{array}{l}1,2, \cdots, N+1 \\ \pi_{1}, \pi_{2}, \cdots, \pi_{N+1}\end{array}\right)$ such that

$$
\frac{f_{1}}{g_{\pi_{1}}}=\frac{f_{2}}{g_{\pi_{2}}}=\cdots=\frac{f_{N+1}}{g_{\pi_{N+1}}} .
$$

Therefore, $f$ and $g$ are related as $L \cdot g=f$ with a projective transformation

$$
L: w_{i}^{\prime}=w_{\pi_{i}} \quad 1 \leqq i \leqq N+1 .
$$

Let us prove Theorem II for the general case. Let $f, g$ be meromorphic maps which satisfy the conditions as in Theorem II, where we assume $f(0), g(0) \notin H_{N+2}$. Choosing homogeneous coordinates as in (4.1), we take representations $f=f_{1}: f_{2}: \cdots: f_{N+1}$ and $g=g_{1}: g_{2}: \cdots: g_{N+1}$ with nowhere zero holomorphic functions $f_{1}, f_{2}, \cdots, f_{N+1}, g_{1}, g_{2}, \cdots, g_{N+1}$. For any $a=\left(a_{1}, a_{2}, \cdots, a_{N+1}\right) \in C^{N+1}-\{0\}$, we consider a holomorphic map $f_{a}$ of $C$ into $P^{N}(C)$ defined as 


$$
f_{a}(z)=f_{1}(a z): f_{2}(a z): \cdots: f_{N+1}(a z) \quad(z \in C)
$$

where $a z=\left(a_{1} z, a_{2} z, \cdots, a_{N+1} z\right)$. And, we define a map $g_{a}: C \rightarrow P^{N}(C)$ similarly by $g$. Then, the following fact is valid.

LEMMA 4.3. Let $E$ be the set of all $a \in C^{n}-\{0\}$ such that $\nu\left(f_{a}, H_{N+2}\right)(z)$ $\neq \nu\left(f, H_{N+2}\right)(a z)$ or $\nu\left(g_{a}, H_{N+2}\right)(z) \neq \nu\left(g, H_{N+2}\right)(a z)$ for some $z$. Then, for the canonical map $\varpi:\left(z_{1}, \cdots, z_{n}\right) \in C^{n}-\{0\} \mapsto z_{1}: \cdots: z_{n} \in P^{n-1}(\mathrm{C})$, the set $\varpi(E)$ is nowhere dense in $P^{n-1}(C)$.

For the proof, see e.g., [3], Proposition 2.7, p. 275.

Let $S_{N+1}$ be the set of all permutations of indices $1,2, \cdots, N+1$. By $L_{\pi}$ we denote the projective linear transformation of $P^{N}(C)$ defined as

$$
L_{\pi}: w_{i}^{\prime}=w_{\pi_{i}} \quad(1 \leqq i \leqq N+1)
$$

for each $\pi=\left(\begin{array}{l}1,2, \cdots, N+1 \\ \pi_{1}, \pi_{2}, \cdots, \pi_{N+1}\end{array}\right) \in S_{N+1}$. For any $a$ in $C^{n}-(E \cup\{0\})$, since $f_{a}$ and $g_{a}$ satisfy the assumptions of Theorem II as holomorphic maps of $C$ into $P^{N}(C)$, applying Theorem II for the case $n=1$, we can conclude that $L_{\pi} \cdot g_{a}=f_{a}$ for some $\pi \in S_{N+1}$. Let $F_{\pi}$ be the set of all points $a$ in $C^{n}-(E \cup\{0\})$ such that $L_{\pi} \cdot g_{a}=f_{a}$. Then, $C^{n}-(E \cup\{0\})=\bigcup_{\pi \in S_{N+1}} F_{\pi}$. Each $F_{\pi}$ is an analytic subset of $C^{n}-(E \cup\{0\})$. In this situation, it can be easily seen that $F_{\pi_{0}}=C^{n}-(E \cup\{0\})$ for some $\pi_{0}$. This shows that Theorem II is also true for the case $n \geqq 2$.

We shall prove next Theorem I. Let $f, g$ be algebraically nondegenerate meromorphic maps of $C^{n}$ into $P^{N}(C)$ such that $\nu\left(f, H_{\imath}\right)=$ $\nu\left(g, H_{i}\right)=0$ for $i=1, \cdots, N+1$ and

$$
\min \left(\nu\left(f, H_{\jmath}\right), N\right)=\min \left(\nu\left(g, H_{\jmath}\right), N\right)
$$

for $j=N+2, \cdots, 2 N+3$. Apply Theorem II to $N+2$ hyperplanes $H_{1}, H_{2}, \cdots, H_{N+1}$ and $H_{i}$ for each $i=N+2, \cdots, 2 N+3$. There is a projective linear transformation $L_{i}$ such that $L_{i} \cdot g=f$ and $L_{i}$ permutes hyperplanes $H_{1}, \cdots, H_{N+1}$ and fixes $H_{i}$. By the assumption of nondegeneracy, we have easily $L:=L_{N+2}=\cdots=L_{2 N+3}$. This implies that $L$ fixes $N+2$ hyperplanes $H_{N+2}, \cdots, H_{2 N+3}$ in general position. It follows that $L=$ identity and so $f=g$, which completes the proof of Theorem I. 


\section{REFERENCES}

[1] E. Borel, Sur les zéros des fonctions entières, Acta Math., 20 (1897), 357-396.

[2] H. Cartan, Sur les zéros des combinaisons linéaires de $p$ fonctions holomorphes. données, Mathematica, 7 (1933), 5-31.

[ 3 ] H. Fujimoto, On meromorphic maps into the complex projective space, J. Math. Soc. Japan, 26 (1974) , 272-288.

[4] - The uniqueness problem of meromorphic maps into the complex projective space, Nagoya Math. J., 58 (1975), 1-23.

[5] — A uniqueness theorem of algebraically non-degenerate meromorphic maps into $P^{N}(C)$, Nagoya Math. J., 64 (1976), 117-147.

[6] - Remarks to the uniqueness problem of meromorphic maps into $P^{N}(C), \mathrm{I}$, Nagoya Math. J., 71 (1978), 13-24.

[ 7 ] R. Nevanlinna, Einige Eindeutigkeitssätze in der Theorie der meromorphen Funktionen, Acta Math., 48 (1926), 367-391.

[8] G. Pólya, Bestimmung einer ganzen Funktionen endlichen Geschlechts durch viererlei Stellen, Math. Tidskrift, B. København 1921, 16-21.

Nagoya University 